\title{
Triplet pairing due to spin-orbit-assisted electron-phonon coupling
}

\author{
Vladimir I. Fal'ko ${ }^{1}$ and Boris N. Narozhny ${ }^{2}$ \\ ${ }^{1}$ Physics Department, Lancaster University, Lancaster LA1 4YB, United Kingdom \\ ${ }^{2}$ The Abdus Salam ICTP, Strada Costiera 11, Trieste I-34100, Italy
}

(Received 11 June 2006; published 17 July 2006)

\begin{abstract}
We propose a microscopic mechanism for spin-dependent attraction in a metal leading to triplet pairing, which is caused by spin-orbit-assisted coupling between electrons and optical phonons in crystals with complex unit cells. Using two examples of crystals with either a cubic or a layered square lattice, we show that spin-orbit-assisted electron-phonon coupling generates triplet pairing with uniquely predetermined tensor structure of the $p$-wave order parameter.
\end{abstract}

DOI: 10.1103/PhysRevB.74.012501

PACS number(s): 74.20.Fg, 74.20.Rp, 74.25.Kc, 74.70.Pq

Spin-triplet superconductivity has been found in several materials including $\mathrm{Sr}_{2} \mathrm{RuO}_{4}$ (Ref. 1) and uranium-based compounds $\left(\mathrm{UPt}_{3}, \mathrm{UBe}_{13}, \mathrm{UGe}_{2}, \mathrm{URuGe}\right){ }^{2-5}$ While the group-theoretical phenomenology of the triplet state has been explored $^{6,7}$ and tables listing all possible classes of $p$-wave states permitted, in principle, for each crystalline symmetry can be found in review articles, ${ }^{1,2,8-10}$ the origin of the microscopic mechanism of the triplet pairing leading to a particular structure of the order parameter in each of these materials and its relation to the band structure still remain open to debate. ${ }^{1-4,11-21}$ The majority of existing models ${ }^{11-17}$ attribute the nature of the $e-e$ attraction in the triplet Cooper channel to an interaction mediated by either ferro- or antiferromagnetic fluctuations (yet to be observed experimentally in materials with signatures of triplet pairing ${ }^{2-5}$ ). Others discuss a spin-independent nonlocal interaction between electrons ${ }^{18,19}$ (including that mediated by phonons in the conventional BSC scheme ${ }^{20}$ ). The latter models can explain the stability of phases with the experimentally identified structure of the order parameter ${ }^{2-5}$ only after imposing spin-orbit (SO) coupling that is linear in the electron spin operator onto the material band structure. ${ }^{21}$ Coupling between spin and orbital degrees of freedom may be relevant ${ }^{22-24}$ for pairing in the inversion-asymmetric $\mathrm{CePt}_{3} \mathrm{Si}^{25}$ However, most of the known triplet superconductors (such as $\mathrm{Sr}_{2} \mathrm{RuO}_{4}$ ) are inversion symmetric, and $\mathrm{SO}$ coupling terms in their band structure that contain the electron spin- $\frac{1}{2}$ operator in linear order are forbidden by the inversion symmetry of the lattices. ${ }^{26,27}$

In this Brief Report, we propose an alternative mechanism of formation of triplet pairs, in which the effective spindependent electron-electron attraction is mediated by spinorbit-assisted electron-phonon (e-ph) (SOEP) coupling. The latter is specific to metals with complex unit cells and strong spin-orbit coupling in constituent heavy atoms. The proposed mechanism is present in both center-symmetric and asymmetric crystals, since sublattice displacements break inversion symmetry, thus enabling atomic spin-orbit coupling to produce a spin-dependent interaction between electrons and optical phonons. Below, we show that the SOEP coupling can, in principle, generate spin-triplet pairing. Moreover, we find that the tensor structure of the order parameter of pairs coupled via SOEP interaction is uniquely determined by the polarization of the most strongly coupled optical phonon mode for each particular lattice. Since SOEP coupling has not been discussed in the context of superconductivity before, ${ }^{1,2,8-10}$ here we demonstrate how this peculiar pairing mechanism determines the symmetry of triplet pairs for the two simplest examples: (i) a cubic lattice with two atoms in the unit cell and a spherical Fermi surface and (ii) a layered structure with in-plane square symmetry and a cylindrical Fermi surface.

In a center-symmetric crystal with a multicomponent unit cell a spin-orbit-assisted electron-phonon coupling arises from a spin-dependent envelope between orbitals belonging to atoms from different sublattices and mutually displaced by the optical phonon vibration (thus locally breaking the inversion symmetry of the unit cell). For a cubic center-symmetric two-component lattice the electron spin coupling to the inversion-symmetry-breaking sublattice displacement $\mathbf{w}$ can be described using the Rashba-type ${ }^{27,28}$ invariant

$$
\hat{V}_{c}=(\alpha / 2)(\mathbf{w} \cdot[\hat{\mathbf{p}} \times \hat{\boldsymbol{\sigma}}]+[\hat{\mathbf{p}} \times \hat{\boldsymbol{\sigma}}] \cdot \mathbf{w}) .
$$

In the second-quantized form, this can be represented as

$$
\begin{gathered}
\hat{\mathcal{V}}=\sum_{\mathbf{p}, \mathbf{q}, \zeta} V_{\mu \nu}^{\zeta}(\mathbf{p}, \mathbf{q}) \hat{f}_{\mu \mathbf{p}+\mathbf{q} / 2}^{\dagger} \hat{f}_{\nu \mathbf{p}-\mathbf{q} / 2}\left(\hat{a}_{\mathbf{q} \zeta}+\hat{a}_{-\mathbf{q} \zeta}^{\dagger}\right), \\
\hat{V}_{c}^{\zeta}=\left(\alpha / \sqrt{2 \omega_{\zeta} m_{\zeta}}\right) \mathbf{l}_{\zeta} \cdot[\mathbf{p} \times \hat{\boldsymbol{\sigma}}] .
\end{gathered}
$$

Here, $\hat{a}^{\dagger}(\hat{a})$ and $\hat{f}^{\dagger}(\hat{f})$ stand for creation (annihilation) operators of phonons and electrons, $\boldsymbol{l}_{\zeta}, \omega_{\zeta}$, and $m_{\zeta}$ are the polarization vector, frequency, and reduced mass of the optical phonon $\zeta$, the indices $\mu, \nu$ identify the electron in the Kramers doublet for each momentum (spin for a simple conduction band), and the parameter $\alpha$ is a material constant. In the cubic crystal, we neglect the small difference between the LO and TO phonon frequencies; thus $\omega_{\zeta} \approx \omega_{o}$. In a less symmetric crystal, $\hat{V}^{\zeta}=\sum_{k=x, y, z} \hat{\sigma}^{k} A_{k}^{\zeta}(\mathbf{p}, \mathbf{q})$, where $A_{k}^{\zeta}(\mathbf{p},-\mathbf{q})=A_{k}^{\zeta}(\mathbf{p}, \mathbf{q})$ and $A_{k}^{\zeta}(-\mathbf{p}, \mathbf{q})=-A_{k}^{\zeta}(\mathbf{p}, \mathbf{q})$; thus, $\hat{V}^{\zeta}(-\mathbf{p}, \mathbf{q})=-\hat{V}^{\zeta}(\mathbf{p}, \mathbf{q}), \hat{V}^{\zeta}(\mathbf{p},-\mathbf{q})=\hat{V}^{\zeta}(\mathbf{p}, \mathbf{q})$. For example, in a layered crystal with a square lattice where we neglect the electron motion perpendicular to the $x-y$ planes, we take into account two invariants analogous to Eq. (1), $\alpha_{\perp} w^{z}\left(p^{x} \hat{\sigma}^{y}-p^{y} \hat{\sigma}^{x}\right)$ and $\alpha_{\|} \hat{\sigma}^{z}\left(w^{x} p^{y}-w^{y} p^{x}\right)$, to distinguish between the electron coupling to optical phonons polarized 
perpendicular to $\left(\zeta=z ; \omega_{z}=\omega_{\perp}\right)$ and in the plane of the layers $\left(\zeta=x, y ; \omega_{x, y}=\omega_{\|}\right)$:

$$
\hat{V}_{\mathrm{sq}}^{z}=\frac{\alpha_{\perp}\left(p^{x} \hat{\boldsymbol{\sigma}}^{y}-p^{y} \hat{\boldsymbol{\sigma}}^{x}\right)}{\sqrt{2 \omega_{\perp} m_{\perp}}}, \quad \hat{V}_{\mathrm{sq}}^{x(y)}=\frac{ \pm \alpha_{\|} p^{y(x)} \hat{\boldsymbol{\sigma}}^{z}}{\sqrt{2 \omega_{\|} m_{\|}}} .
$$

The SOEP coupling in Eq. (2) results in an effective interaction between electrons on the opposite sides of the Fermi surface,

$$
\begin{gathered}
\mathcal{U}=-\sum_{\mathbf{p}_{1}, \mathbf{p}_{2}} M_{\gamma \delta}^{\alpha \beta} \hat{f}_{\alpha \mathbf{p}_{1}}^{\dagger} \hat{f}_{\gamma-\mathbf{p}_{1}}^{\dagger} \hat{f}_{\delta-\mathbf{p}_{2}} \hat{f}_{\beta \mathbf{p}_{2}}, \\
M_{\gamma \delta}^{\alpha \beta}=\sum_{\zeta} \frac{\omega_{\zeta} V_{\alpha \beta}^{\zeta}\left(\mathbf{p}_{1}+\mathbf{p}_{2}\right) V_{\gamma \delta}^{\zeta}\left(-\mathbf{p}_{2}-\mathbf{p}_{1}\right)}{\omega_{\zeta}^{2}-\left(\varepsilon_{\mathbf{p}_{2}}-\varepsilon_{\mathbf{p}_{1}}\right)^{2}} .
\end{gathered}
$$

In Eq. (5), we neglect the dispersion of optical phonon modes, $\omega_{\zeta}(q) \approx \omega_{\zeta}$, and take into account the quasiparticle states close to the Fermi surface $\left(p=|\mathbf{p}| \approx p_{F}\right)$, with $\left|\varepsilon_{\mathbf{p}_{i}}\right|<\omega_{\zeta}$, where $\varepsilon_{\mathbf{p}}$ is the quasiparticle energy calculated with respect to the Fermi level. The effective $e$-e interaction $\mathcal{U}$ is spin and momentum dependent; therefore, it may allow for attraction in the spin-triplet channel and odd pairing.

To determine what triplet phase can be formed in a metal with the Cooper interaction in Eqs. (4) and (5), we identify Cooper channels in which the $e$-e interaction is attractive. Following the standard procedure, ${ }^{29,30}$ we determine operators of singlet $(\hat{b})$ and triplet $\left(\hat{t}_{s}\right)$ pairs, $\hat{b}(\mathbf{p})=i \sigma_{\mu y}^{y} \hat{f}_{\mu-\mathbf{p}} \hat{f}_{\nu \mathbf{p}}$, $\hat{t}_{s}(\mathbf{p})=i\left(\sigma^{y} \sigma^{s}\right)_{\mu \nu} \hat{f}_{\mu-\mathbf{p}} \hat{f}_{\nu \mathbf{p}}$. In terms of these operators, ${ }^{30-32} \mathcal{U}$ in Eq. (4) can be written down in a separable form,

$$
\mathcal{U}=-\sum_{\mathbf{p}_{1} \mathbf{p}_{2}}\left[T^{S_{1} s_{2}} t_{s_{1}}^{\dagger}\left(\mathbf{p}_{1}\right) t_{s_{2}}\left(\mathbf{p}_{2}\right)+C \hat{b}^{\dagger}\left(\mathbf{p}_{1}\right) \hat{b}\left(\mathbf{p}_{2}\right)\right]
$$

which includes the interaction both in the singlet channel (with $C=M_{\gamma \delta}^{\alpha \beta} \sigma_{\gamma \alpha}^{y} \sigma_{\beta \delta}^{y}$ ) and in the triplet channel, with

$$
T^{s_{1} s_{2}}=M_{\gamma \delta}^{\alpha \beta}\left(\hat{\boldsymbol{\sigma}}^{y} \hat{\boldsymbol{\sigma}}^{s_{1}}\right)_{\gamma \alpha}\left(\hat{\boldsymbol{\sigma}}^{s_{2}} \boldsymbol{\sigma}^{y}\right)_{\beta \delta} .
$$

Triplet pairs can be characterized by quantum numbers related to irreducible representations of the corresponding symmetry group of a crystal, ${ }^{6-9,30,32}$ where the $p$-wave order parameter is a tensor,

$$
B_{m s}=\sum_{\mathbf{p}} n_{m}\left\langle\hat{t}_{s}(\mathbf{p})\right\rangle, \quad \mathbf{n}=\frac{\mathbf{p}}{p} .
$$

(i) In the case of a model metal with a cubic lattice and a spherical Fermi surface, the symmetry is the full rotational group $\mathrm{SO}_{3}$. The nature of the SOEP interaction responsible for the pair formation assumes that the spin and orbital degrees of freedom of the Cooper pair are not independent, so that its total spin $S=1$ and orbital angular momentum, $L=1$ sum into the total angular momentum, $J=0,1,2$. The tensor structure of the order parameter for these values of $J$ is the following: $B_{m s}^{J=0}=\delta^{n s} D e^{i \phi}$, where $B$ is a scalar; $B_{m s}^{J=1}=\epsilon^{m s l} D_{l}$, where $\mathbf{D}$ is a complex vector; and $B_{m s}^{J=2}$ is a traceless symmetric tensor. ${ }^{6-9,30,32}$
Interaction in Eq. (6) can be decomposed into three channels corresponding to different angular momenta of the pair. Using the relation of the matrix $M_{\gamma \delta}^{\alpha \beta}$ with the $e$-ph coupling $\hat{V}_{c}^{\zeta}$ in Eq. (2), we find that

$$
\begin{gathered}
T^{s_{1} s_{2}}=g_{\mathrm{so}} \sum_{m_{1}, m_{2}} n_{1}^{m_{1}} n_{2}^{m_{2}} G_{m_{1} m_{2}}^{s_{1} s_{2}}, \\
G_{m_{1} m_{2}}^{s_{1} s_{2}}=\varkappa_{0} \delta^{n_{1} s_{1}} \delta^{m_{2} s_{2}}+\varkappa_{1} \epsilon^{m_{1} s_{1} l} \epsilon^{m_{2} s_{2} l} \\
+\varkappa_{2}\left[\delta^{m_{1} m_{2}} \delta^{s_{1} s_{2}}+\delta^{n_{1} s_{2}} \delta^{m_{2} s_{1}}-\frac{2}{3} \delta^{n_{1} s_{1}} \delta^{m_{2} s_{2}}\right] .
\end{gathered}
$$

The first, second, and third terms in the matrix $G_{m_{1} m_{2}}^{s_{1} s_{2}}$ are products of projectors onto the $J=0,1$, and 2 states, respectively. Interaction in each $J$ channel is described by the corresponding constant,

$$
g_{J}=\varkappa_{J} g_{\mathrm{so}}, \quad g_{\mathrm{so}} \sim\left(\alpha / \omega_{o}\right)^{2} p_{F}^{2} / 2 m_{o},
$$

where the scale of the interaction depends on the strength of the original $e$-ph interaction for electrons near the Fermi surface, and on the frequency $\omega_{o}$ and reduced mass $m_{o}$ of the optical phonon (here we disregard the small difference between longitudinal and transverse optical phonon modes in a cubic crystal). Interaction in channels with different $J$ 's differs by the numerical factors

$$
x_{0}=-\frac{4}{3}, \quad x_{1}=\frac{1}{2}, \quad x_{2}=-\frac{1}{2} .
$$

According to Eq. (6), the positive sign of $g_{J}$ corresponds to attraction. Since only one of the effective coupling constants $g_{J}=g_{\text {so }} \varkappa_{J}$ is positive, we conclude that in the cubic system Cooper interaction due to the spin-orbit-assisted $e$-ph coupling leads to a condensate of pairs with the total angular momentum $J=1$.

By selecting a pairing channel with $J=1$ we do not fully determine the order parameter $B_{m s}$ in the lowest-energy triplet state yet. In $B_{m s}^{J=1}=\epsilon^{m s l} D_{l}, \mathbf{D}=D e^{i \phi}\left(\boldsymbol{l}+i \boldsymbol{l}^{\prime}\right) / \sqrt{\boldsymbol{l}^{2}+\left(\boldsymbol{l}^{\prime}\right)^{2}}$ is a complex vector ${ }^{32}\left[\boldsymbol{l}\right.$ and $\boldsymbol{l}^{\prime}$ are two perpendicular vectors, $\left.l^{2} \geqslant\left(l^{\prime}\right)^{2}\right]$. Following Refs. 6-9, 30, and 32 we find a favorable state by minimizing the condensate energy

$$
\mathcal{E}=\sum_{\mathbf{p} \mu}\left(\left|\varepsilon_{\mathbf{p}}\right|-E_{\mathbf{p} \mu}\right)+\sum_{m s} g\left|B_{m s}\right|^{2}
$$

as a function of $\mathbf{D}$, which includes finding the relative size of two vectors $\boldsymbol{l}$ and $\boldsymbol{l}^{\prime}$ and the absolute value of $D$. The expression for $\mathcal{E}$ in Eq. (11) has been obtained using the HubbardStratonovich transformation which splits the $e$-e interaction in Eq. (6) using the field $B_{m s}$ and then by integrating out fermionic degrees of freedom. In Eq. (11), the spectrum of excitations $^{30,32}$ in the phase with order parameter $B_{m s}$ is described by

$$
E_{\mathbf{p} \mu}=\sqrt{\varepsilon_{\mathbf{p}}^{2}+\left|\mathbf{d}_{\mathbf{p}}\right|^{2}-\mu\left|\left[\mathbf{d}_{\mathbf{p}} \times \mathbf{d}_{\mathbf{p}}^{*}\right]\right|},
$$

where three components of the complex vector $\mathbf{d}_{\mathbf{p}}$ are defined as 
TABLE I. $J=1$ phases due to SOEP interaction in a metal with spherical Fermi surface and cubic lattice.

\begin{tabular}{ccc}
\hline \hline Vector $\mathbf{D}$ & Mean-field $D$ & Energy $\mathcal{E}$ \\
\hline $\boldsymbol{l} e^{i \phi} D$ & $\left(2 \omega_{o} / g\right) e^{-3 / g \nu}$ & $-\nu g^{2} D^{2} / 3$ \\
$\frac{\boldsymbol{l}+i \boldsymbol{l}^{\prime}}{\sqrt{2}} e^{i \phi} D$ & $\left(\sqrt{2} \omega_{o} / g\right) e^{-6 / g \nu}$ & $-\nu g^{2} D^{2} / 6$ \\
\hline \hline
\end{tabular}

$$
d_{\mathbf{p}}^{s}=g \sum_{m} n_{m} B_{m s}
$$

The vector product $i \mathbf{d}_{\mathbf{p}} \times \mathbf{d}_{\mathbf{p}}^{*}$ determines the spin quantization axis and spin splitting for Bogoliubov quasiparticles for each direction $\mathbf{n}=\mathbf{p} / p$ on the Fermi sphere and $\mu= \pm 1$ denotes the polarization of the quasiparticle spin with respect to such an axis. In Eqs. (11) and (12), $\varepsilon_{\mathbf{p}}$ is the quasiparticle energy in the normal state calculated with respect to the Fermi level, and $g$ is the interaction constant in the attraction channel (here, $g=g_{1} \equiv \varkappa_{1} g_{\text {so }}$ ).

In the context of pairing with $J=1$ and $B_{m s}^{J=1}=\epsilon^{m s l} D_{l}$, two possible extrema of the function $\mathcal{E}$ can be found using the analysis of the degeneracy space of the order parameter. ${ }^{32}$ For fixed $|\mathbf{D}|=D, \mathcal{E}(\mathbf{D})$ takes extremal values on vectors $\mathbf{D}$ that cover minimal surfaces under the application of $\mathrm{SO}_{3}$ rotations. Two such possibilities are $\mathbf{D}_{0}=l \mathbf{e}^{i \phi} D$ where $\boldsymbol{l}$ is a real unit vector (that is, $\boldsymbol{l}^{\prime}=0$ ), and $\boldsymbol{D}_{1}=\frac{\boldsymbol{l}+i \boldsymbol{l}^{\prime}}{\sqrt{2}} \mathbf{e}^{\mathbf{i} \phi} D$, where $\boldsymbol{l}$ and $l^{\prime}$ are two perpendicular unit vectors. For each of these two cases we find the value of $D$ by minimizing the ground-state energy $\mathcal{E}$ in Eq. (11). The results ${ }^{33}$ are listed in Table I indicating that the phase with $\boldsymbol{D}_{0}=\boldsymbol{l} e^{i \phi} D$ and an anisotropic gap vanishing along the direction $\boldsymbol{l}$ (since $\boldsymbol{d}=g D[\boldsymbol{n} \times \boldsymbol{l}]$ ) is energetically favorable.

(ii) In a layered material with a square-symmetric lattice, the SOEP interaction is also able to generate triplet pairing with definite phases. For simplicity (and with reference to an almost cylindrical $\gamma$ sheet in the Fermi surface of ${ }^{1} \mathrm{Sr}_{2} \mathrm{RuO}_{4}$ ) we consider below a metal with a cylindrical Fermi surface. As in BCS theory, the effective attraction $\mathcal{U}$ in Eqs. (4) and (6) is pointlike, so that only electrons from the same layer can form a pair. Thus, we shall consider only twodimensional order parameters (the interlayer tunneling can be treated using the Lawrence-Doniach approach ${ }^{29}$ ). Following Refs. 6-9 and 30 triplet $p$-wave pairs can be characterized by the component $J_{z}$ of the total angular momentum in the direction perpendicular to the layers, which is the sum of the projection $L_{z}= \pm 1$ of their orbital angular momentum and
$S_{z}=0, \pm 1$ of the pair spin. For $J_{z}= \pm 1$ and \pm 2 , the quantum number $J_{z}$ fully determines the order parameter, whereas for $J_{z}=0$, pairs should be additionally classified according to whether they are symmetric $(s)$ or antisymmetric $(a)$ with respect to in-plane $x \rightleftarrows y$ reflection, for which we reserve the notations $J_{z}=0^{s}$ and $J_{z}=0^{a}$, respectively. The second line in Table II summarizes the tensor structure $B_{m s}=P_{m s}^{J_{z}} e^{i \phi} D$ of the order parameter for phases with $J_{z}=0^{s}, 0^{a}, \pm 1, \pm 2$, where the matrices $P_{m s}^{J_{z}}$ form the orthogonal basis of irreducible representations of $J_{z}$, and where $D^{2}=\Sigma_{m s}\left|B_{m s}\right|^{2}$.

Using the matrices $P^{J_{z}}$ the effective interaction $\hat{\mathcal{U}}$ can be written down in the separable form Eq. (6) with

$$
\begin{gathered}
T^{s_{1} s_{2}}=\sum_{m_{1} m_{2} J_{z}}\left(\varkappa_{J_{z}} g_{\text {so }}^{\perp}+\tilde{x}_{J_{z}} g_{\text {so }}^{\|}\right) n_{1}^{m_{1}} n_{2}^{m_{2}} P_{s_{1} m_{1}}^{J_{z}} P_{s_{2} m_{2}}^{J^{*}}, \\
g_{\text {so }}^{\zeta} \sim\left(\alpha_{\zeta} / \omega_{\zeta}\right)^{2} p_{F}^{2} / 2 m_{\zeta}, \quad \zeta=\perp, \|,
\end{gathered}
$$

which is determined by couplings (3) to optical phonons polarized across $(\zeta=\perp)$ and parallel to $(\zeta=\|)$ the layer. The values of the coefficients $\varkappa_{J_{z}}$ and $\tilde{\varkappa}_{J_{z}}$ are listed in Table II, which shows that positive coupling constants (determining the attraction in the corresponding Cooper channel) may appear only for electron pairs in the following channels: (a) $J_{z}=0^{a}$ where $g_{0^{a}}=\varkappa_{0^{a}} g_{\text {so }}^{\perp}+\tilde{\varkappa}_{0} g_{\text {so }}^{\|}=2 g_{\text {so }}^{\perp}-g_{\text {so }}^{\|}$, and (b) $J_{z}= \pm 1$ where $g_{ \pm 1}=\varkappa_{ \pm 1} g_{\mathrm{so}}^{\perp}+\tilde{\varkappa}_{ \pm 1} g_{\mathrm{so}}^{\|}=g_{\mathrm{so}}^{\|}-g_{\mathrm{so}}^{\perp}$. This indicates that, within the proposed mechanism, only two triplet phases are possible.

To find which phase is realized we need to compare the relative strengths of the electron coupling to the in- and outof-plane sublattice vibrations. If $g_{\text {so }}^{\|}<3 / 2 g_{\text {so }}^{\perp}$, so that $g_{0^{a}}>g_{ \pm 1}$, the ground state with $J_{z}=0^{a}$ is realized. This phase is characterized by the vector order parameter $\mathbf{d}$ and an isotropic gap,

$$
\mathbf{d}_{0^{a}}=\frac{g D}{\sqrt{2}}\left(\begin{array}{c}
n^{y} \\
-n^{x} \\
0
\end{array}\right),
$$

$$
E_{\mathbf{p}}=\sqrt{\varepsilon_{\mathbf{p}}^{2}+\Delta^{2}}, \quad \Delta=2 \omega_{\perp} e^{-2 / v g}, \quad g=2 g_{\text {so }}^{\perp}-g_{\text {so }}^{\|} .
$$

Here ${ }^{33} D=2^{3 / 2}\left(\omega_{\perp} / g\right) e^{-2 / \nu g}$ was found from minimizing $\mathcal{E}$ in Eq. (11) with respect to $D$.

If $g_{\text {so }}^{\|}>\frac{3}{2} g_{\text {so }}^{\perp}$, so that $g_{ \pm 1}>g_{0^{a}}$ [in terms of microscopic

\begin{tabular}{|c|c|c|c|}
\hline \multicolumn{4}{|c|}{$J_{z}$} \\
\hline \pm 2 & $\pm 1(\mathrm{ABM})$ & $0^{s}$ & $0^{a}$ \\
\hline$P_{s m}^{ \pm 2}=\frac{1}{2}\left(\delta^{m x} \pm i \delta^{m y}\right)\left(\delta^{s x} \pm i \delta^{s y}\right)$ & $P_{s m}^{ \pm 1}=\frac{1}{\sqrt{2}}\left(\delta^{m x} \pm i \delta^{m y}\right) \delta^{s z}$ & $P_{s m}^{0^{s}}=\frac{1}{\sqrt{2}}\left(\delta^{m x} \delta^{s x}+\delta^{m y} \delta^{s y}\right)$ & $P_{s m}^{0^{a}}=\frac{1}{\sqrt{2}}\left(\delta^{m y} \delta^{s x}-\delta^{m x} \delta^{s y}\right)$ \\
\hline$x_{ \pm 2}=0$ & $x_{ \pm 1}=-1$ & $x_{0^{s}}=-2$ & $x_{0^{a}}=2$ \\
\hline$\tilde{\varkappa}_{ \pm 2}=-1$ & $\tilde{x}_{ \pm 1}=1$ & $\tilde{\varkappa}_{0^{s}}=-1$ & $\tilde{\varkappa}_{0^{a}}=-1$ \\
\hline
\end{tabular}
parameters, for a stronger coupling to the in-plane sublattice displacement, $\left.\quad \alpha_{\|} /\left(\sqrt{m_{\|}} \omega_{\|}\right)>\sqrt{\frac{3}{2}} \alpha_{\perp} /\left(\sqrt{m_{\perp}} \omega_{\perp}\right)\right]$, then the

TABLE II. Triplet pairing order parameter for electrons with cylindrical Fermi surface in a layered material with square lattice and the interaction constants in the corresponding $J_{z}$ channels due to the spin-orbit-assisted $e$-ph coupling. 
ground state belongs to the phase with $J_{z}= \pm 1$ which is similar to the Anderson-Brinkmann-Morel (ABM) phase pr,6-9,30 $^{1,0}$ with a circularly isotropic gap:

$$
\begin{gathered}
\mathbf{d}_{ \pm 1}=\frac{g D}{\sqrt{2}}\left(\begin{array}{c}
0 \\
0 \\
n^{x} \pm i n^{y}
\end{array}\right), \\
E_{\mathbf{p}}=\sqrt{\varepsilon_{\mathbf{p}}^{2}+\Delta^{2}}, \quad \Delta=2 \omega_{\|} e^{-2 / v g}, \quad g=2 g_{\text {so }}^{\|}-g_{\text {so }}^{\perp} .
\end{gathered}
$$

In conclusion, we propose a mechanism of triplet pairing in center-symmetric crystals with a complex unit cell based upon spin-orbit-assisted $e$-ph coupling. We show that the SOEP interaction generates phases with a definite $p$-wave order parameter uniquely determined by crystal symmetry and the polarization of the most strongly coupled optical phonon mode. In a layered metal where conducting planes have square lattice structure, the proposed mechanism of triplet pairing leads to phases described by Eqs. (14) and (15), one of which (the ABM phase) is now being considered $^{1}$ as a plausible candidate for the superconditing order parameter in $\mathrm{Sr}_{2} \mathrm{RuO}_{4}$ (which has a layered perovskite structure with body-centered tetragonal group symmetry). On one hand, the latter coincidence may be purely accidental, so that a detailed analysis of the phonon spectrum and SOEP interaction strength for different sheets of the Fermi surface in strontium ruthenate is needed. On the other hand, a pairing mechanism via the spin-orbit-assisted $e$-ph coupling would explain the isotopic shift of $T_{c}$ recently observed in $\mathrm{Sr}_{2} \mathrm{RuO}_{4}$ using oxygen isotopes. ${ }^{34,35}$

The authors thank P. Fulde, B. Gyorffy, R. Joynt, A. Mackenzie, and E. Tosatti for discussions and comments. This work was supported by EPSRC and EC Grant No. NMP2-CT2003-505587 "SFINX."
${ }^{1}$ A. P. Mackenzie and Y. Maeno, Rev. Mod. Phys. 75, 657 (2003), and references therein.

${ }^{2}$ R. Joynt and L. Taillefer, Rev. Mod. Phys. 74, 235 (2002).

${ }^{3}$ S. S. Saxena et al., Nature (London) 406, 587 (2000).

${ }^{4}$ H. R. Ott et al., Phys. Rev. Lett. 52, 1915 (1984).

${ }^{5}$ F. Hardy and A. D. Huxley, Phys. Rev. Lett. 94, 247006 (2005).

${ }^{6}$ G. E. Volovik and L. P. Gorkov, Zh. Eksp. Teor. Fiz. 88, 1412 (1985) [Sov. Phys. JETP 61, 843 (1985)].

${ }^{7}$ E. I. Blount, Phys. Rev. B 32, 2935 (1985).

${ }^{8}$ R. Joynt et al., Phys. Rev. B 42, 2014 (1990).

${ }^{9}$ J. F. Annett, Adv. Phys. 39, 83 (1990); I. Eremin et al., Ann. Phys. 13, 149 (2004).

${ }^{10}$ M. Sigrist and K. Ueda, Rev. Mod. Phys. 63, 239 (1991).

${ }^{11}$ M. Sigrist and T. M. Rice, J. Phys.: Condens. Matter 7, L643 (1995); D. F. Agterberg, T. M. Rice, and M. Sigrist, Phys. Rev. Lett. 78, 3374 (1997); T. M. Rice, Nature (London) 396, 627 (1998)

${ }^{12}$ M. E. Zhitomirsky and T. M. Rice, Phys. Rev. Lett. 87, 057001 (2001).

${ }^{13}$ D. L. Cox and M. B. Maple, Phys. Today 48 (2), 32 (1995).

${ }^{14}$ R. Roussev and A. J. Millis, Phys. Rev. B 63, 140504 (2001); Z. Wang, W. Mao, and K. Bedell, Phys. Rev. Lett. 87, 257001 (2001).

${ }^{15}$ I. I. Mazin and D. J. Singh, Phys. Rev. Lett. 79, 733 (1997); Phys. Rev. Lett. 82, 4324 (1999).

${ }^{16}$ A. B. Shick and W. E. Pickett, Phys. Rev. Lett. 86, 300 (2001).

${ }^{17}$ T. R. Kirkpatrick and D. Belitz, Phys. Rev. Lett. 92, 037001 (2004).

${ }^{18}$ S. Koikegami, Y. Yoshida, and T. Yanagisawa, Phys. Rev. B 67, 134517 (2003).

${ }^{19}$ J. Annett et al., Phys. Rev. B 66, 134514 (2002).

${ }^{20}$ I. F. Foulkes and B. L. Gyorffy, Phys. Rev. B 15, 1395 (1977); H. Shimahara and M. Kohmoto, ibid. 65, 174502 (2002).
${ }^{21}$ J. Annett et al., cond-mat/0411169 (unpublished).

${ }^{22}$ L. P. Gorkov and E. I. Rashba, Phys. Rev. Lett. 87, 037004 (2001).

${ }^{23}$ P. A. Frigeri et al., Phys. Rev. Lett. 92, 097001 (2004).

${ }^{24}$ K. V. Samokhin, E. S. Zijlstra, and S. K. Bose, Phys. Rev. B 69, 094514 (2004).

${ }^{25}$ E. Bauer et al., Phys. Rev. Lett. 92, 027003 (2004).

${ }^{26}$ G. Dresselhaus, Phys. Rev. 100, 580 (1955).

${ }^{27}$ E. I. Rashba, Sov. Phys. Solid State 2, 1109 (1960); Yu. Bychkov and E. I. Rashba, JETP Lett. 39, 78 (1984).

${ }^{28}$ We symmetrize $\hat{V}_{c}$ to take into account noncommutation of the momentum $\hat{\mathbf{p}}$ and the coordinate-dependent $\mathbf{w}(\mathbf{x})$.

${ }^{29}$ J. B. Ketterson and S. N. Song, Superconductivity (Cambridge University Press, Cambridge, UK, 1998).

${ }^{30} \mathrm{P}$. W. Anderson and W. F. Brinkmann, in The Physics of Liquid and Solid Helium, edited by K. H. Bennemann and J. B. Ketterson (Wiley, New York, 1978).

${ }^{31}$ The operator $\hat{b}$ is even in $\mathbf{p}$, while $\hat{t}_{s}$ are odd. The $e$-ph coupling generates an effective $e-e$ interaction in the triplet channel, due to the asymmetry $\hat{V}^{\zeta}(-\mathbf{p}, \mathbf{q})=-\hat{\mathbf{V}}^{\zeta}(\mathbf{p}, \mathbf{q})$ and $\hat{V}^{\zeta}(\mathbf{p},-\mathbf{q})$ $=\hat{\mathbf{V}}^{\zeta}(\mathbf{p}, \mathbf{q})$ of its spin-orbit-assisted part. The latter property is specific to optical phonons. The acoustic $e$-ph coupling has a different symmetry, $\hat{V}^{\zeta}(\mathbf{p},-\mathbf{q})=-\hat{\mathbf{V}}^{\zeta}(\mathbf{p}, \mathbf{q})$.

${ }^{32}$ V. I. Fal'ko and I. S. Shapiro, Zh. Eksp. Teor. Fiz. 91, 1194 (1986) [Sov. Phys. JETP 64, 706 (1986)].

${ }^{33} \nu$ is the Fermi density of states in one spin component.

${ }^{34}$ Z. Q. Mao et al., Phys. Rev. B 63, 144514 (2001).

${ }^{35}$ The sign of the isotope effect observed in Ref. 34 depended on the quality of the structure. The inverse sign of the isotope effect in the purest samples in Ref. 34 may have been the result of pair breaking by the annealing-induced disorder and differently diffusing light and heavy isotopes. 